\title{
An Association Study Between Educational Attainment-Related Genes and Cognitive Functions in Japanese Patients with Schizophrenia
}

\author{
YASUE MIKI*, TOHRU OHNUMA*, HitOKI HIROSE*, KEN NAKAYAMA*, WANYI MAO*, \\ MARIKO NAKADAIRA*, NARIHIRO ORIMO*, HIROKI YAMASHITA*, YUTO TAKEBAYASHI*, \\ ERIKO TANI*, NARIMASA KATSUTA*, SHOHEI NISHIMON*, HEII ARAI* \\ *Department of Psychiatry, Juntendo University Faculty of Medicine, Tokyo, Japan
}

Objective: The present study aimed to investigate the pleiotropic effects of candidate loci identified by genome-wide association studies, how they may function as possible proxy phenotypes for educational attainment, and how they affect clinical symptoms and their detailed psychometrics in Japanese patients with schizophrenia.

Method: Three single-nucleotide polymorphisms (SNPs) (rs6739979, rs11588857, and rs2245901) common in Japanese individuals showing a relationship to both schizophrenia and educational attainments from a previously conducted genome-wide study (Okbay, 2016) were investigated in a case-control study between 640 unrelated Japanese patients with schizophrenia and 640 healthy controls. The relationship between these SNPs and detailed clinical information, including educational attainments and cognitive function from psychometrics, were investigated in these patients.

Results: Results of the present study show that these SNPs are not genetic risk factors for schizophrenia. However, SNP rs2245901 in the 2q32.3 region showed a relationship to declining performance intelligent quotients in schizophrenia patients, as seen from multiple linear regression analysis.

Conclusion: The genetic region at $2 \mathrm{q} 32.3$ may influence the attained education and decline of cognitive function in patients with schizophrenia.

Key words: schizophrenia, 2q32.3, pleiotropy, performance intelligence quotient, educational attainment

\section{Introduction}

Schizophrenia is a debilitating disease with a prevalence of approximately $0.5-1 \%$ within a given population that clinically shows some inheritable features. As such, several studies have investigated the etiology of schizophrenia and conducted genetic analyses to identify specific candidate genes involved in the polygenic heritance of schizophrenia ${ }^{1)}$. Recent genome-wide association studies (GWASs) have shown that certain candidate genetic regions are associated with schizophre- nia (see SZGene section of Schizophrenia Research Forum; http://www.szgene.org/). Detailed psychological assessments, such as the Wechsler Adult Intelligence Scale (WAIS)-III ${ }^{2)}$, the Japanese Adult Reading Test (JART; Japanese version of the National Adult Reading Test for estimating premorbid intelligence) ${ }^{3)}$, and frontal lobe cognitive function tests (e.g., verbal fluency tests and Stroop test ${ }^{4-6)}$, have demonstrated that patients with schizophrenia often display disturbed intelligence and cognitive dysfunctions. These disturbed cognitive functions may also affect some of their

Corresponding author: Tohru Ohnuma

Department of Psychiatry, Juntendo University Faculty of Medicine

2-1-1 Hongo, Bunkyo-ku, Tokyo 113-8421, Japan

TEL \& FAX: +81-35802-1071 E-mail: otoru@juntendo.ac.jp

〔Received Jan. 17, 2019] [Accepted Feb. 15, 2019]

J-STAGE Advance published date: July 24, 2019

Copyright (C) 2019 The Juntendo Medical Society. This is an open access article distributed under the terms of Creative Commons Attribution License (CC BY), which permits unrestricted use, distribution, and reproduction in any medium, provided the original source is properly credited. doi: 10.14789/jmj.2019.65.JMJ19-OA01 
symptoms ${ }^{7)-10)}$.

Interestingly, a GWAS of educational attainment was conducted with a large number of subjects, and the results of this study suggest that three independent single-nucleotide polymorphisms (SNPs) have genome-wide significance (rs9320913, rs11584700, and rs4851266). This genetic information may be useful as a proxy phenotype for educational attainments ${ }^{11}$. Another GWAS identified 74 genome-wide loci significantly associated with educational attainments, and candidate genes from these genomic regions play important roles in the biological pathways involved in neural development ${ }^{12}$. Among these 74 loci, seven loci held particular importance for patients with schizophrenia, and may have pleiotropic effects on both schizophrenia and educational attainment (see recent Extended Data Figure-5 (Q-Q plots for the 74 lead Edu Years SNPs in published GWAS of schizophrenia and additional sheet "3.2 SNP Lookup") ${ }^{12)}$.

The present study aimed to investigate the pleiotropic effects of these candidate loci, how they may function as possible proxy phenotypes for educational attainment, and how they affect clinical symptoms, and their additional detailed psychometrics in Japanese patients with schizophrenia.

\section{Methods}

\section{Participants}

Written informed consent was obtained from all subjects after the procedures had been fully explained. The present study was carried out in compliance with the World Medical Association's Declaration of Helsinki and was approved by the Research Ethical Committees of Juntendo University (2015014).

A case-control genetic association study was performed using 640 unrelated Japanese patients with schizophrenia (312 males, 328 females; mean age 38.1 years, S.D. \pm 11.4 ). All patients met the diagnostic criteria for schizophrenia based on structured clinical interviews from the Diagnostic and Statistical Manual of Mental Disorders-V $(\mathrm{DSM}-\mathrm{V})$. A total of 640 healthy controls (322 males, 318 females; mean age 43.3 years, S.D. \pm 11.9) were also included and examined. Healthy controls did not meet current or past criteria for any Axis I disorder (from the DSM-V). Addition- ally, all participants met the following criteria: (1) no evidence of systemic or neurological diseases, (2) no prior head trauma with loss of consciousness, and (3) no lifetime history of alcohol or substance dependency. Patients and controls were recruited from two geographic regions in Japan: Saitama and Tokyo. The mean age for the patients with schizophrenia was significantly younger than that of the control group (Student's $\mathrm{t}$-test: $\mathrm{t}=6.33, \mathrm{p}<0.001)$. Additionally, the distribution between males and females within the two groups was not significantly different $\left(\chi^{2}=2.11, \mathrm{p}=\right.$ $0.14)$.

\section{SNP selection and genotyping}

Genomic DNA was extracted from peripheral white blood cells using a QIAamp ${ }^{\circledR}$ DNA Blood Maxi kit (Qiagen, Courtaboeuf, France). Among the seven SNPs identified in the previous study as affecting both schizophrenia and educational attainments ${ }^{12}$, allelic frequencies of four of the SNPs were very rare in the Japanese population (minor allele frequency $<0.05$ ); thus, these SNPs did not meet the common-disease common variant criteria. The remaining three SNPs--rs6739979 (2q32), rs11588857 (1q32), and rs2245901 (2q32) (The "rs" notation in front of each SNP represents the identification from the US National Center for Biotechnology Information SNP cluster within the dbSNP database; http://www.ncbi.nlm.nih.gov/ SNP/) -- were analyzed using TaqMan ${ }^{\circledR}$ technology $\left(\right.$ Assay-by-Design $^{\mathrm{TM}}$ ) on an ABI7500 system (Applied Biosystems, Foster City, CA, USA). All probes and primers were designed by the Assayby-Design $^{\mathrm{TM}}$ service provided by Applied Biosystems. Polymerase chain reaction (PCR) was conducted using the standard PCR MasterMix reagent kit in a $4 \mu l$ volume. Additionally, to ensure the quality of the results, we confirmed the SNPs from a few randomly chosen subjects using a direct DNA sequencing method (the TaqMan ${ }^{\circledR}$ method) to check for errors. All genotypes determined via direct sequencing were in agreement with the genotypes obtained from TaqMan ${ }^{\circledR}$ methods for all investigated SNPs. Detailed information regarding the PCR conditions and primer pairs is available upon request. 


\section{Clinical and cognitive assessments}

Clinical variables and in-patient symptoms were assessed in detail by a psychiatrist based on detailed interviews with the patients and their family members. In-patients were also examined using a battery of cognitive assessment tests administered by an expert psychologist. The daily doses of antipsychotics were converted into chlorpromazineequivalent doses ${ }^{13)}$. Clinical symptoms were assessed using the Brief Psychiatric Rating Scale (BPRS), with each item rated on a 7-point scale, as previously described ${ }^{14)}$, and the overall total ratings were compared. The age at the onset of illness was determined as the age at which any schizophrenia symptom described in the diagnostic criteria of DSM-V first appeared and was established on the basis of the patients' medical records and/or by detailed questioning of the patients and their family members.

Mentioned below are the social adjustment assessments and cognitive function batteries, which frequently showed disturbances in patients with schizophrenia that were selected. These assessments were performed after improvement in their severe symptoms present at the time of admission. The social status of each patient was assessed using the Comprehensive Assessment of Symptoms and History (CASH) ${ }^{15)}$ and Modified Premorbid Adjustment Scale (MPAS) ${ }^{16)}$. To assess the patients' present and premorbid intelligence, the Wechsler Adult Intelligence Scale-Revised (WAIS-R) ${ }^{17)}$ and the JART; Japanese version of the National Adult Reading Test) ${ }^{18)}$ were used, respectively. To assess prefrontal cortex cognitive functions, verbal fluency tests ${ }^{19)}$ 20) and the Stroop test ${ }^{6)}{ }^{21}{ }^{22}$ ) were administered as in our previous study ${ }^{23)}$.

\section{Statistical analysis}

Age differences between healthy controls and patients were examined using the two-tailed Student's t-test. Chi-square $\left(\chi^{2}\right)$ tests were used to assess differences in the distribution between males and females. For the case-control association study, Hardy-Weinberg equilibrium (HWE) tests for the SNPs were run using SNPAlyze software, Version 7.0 Pro (Dynacom, Yokohama, Japan). The HWE tests were carried out for all loci in both patients and controls. Differences in genotypic and allelic frequencies were evaluated using $\chi^{2}$ difference tests. All reported p-values are two-tailed. Statistical significance was set on the basis of Bonferroni correction (the probability level of $\mathrm{p}<0.05 / 3$ SNPs $=0.0167$ in each analysis).

Linkage disequilibrium (LD), denoted as D' or $\mathrm{r}^{2}$, was calculated from haplotype frequencies via an expectation-maximization algorithm calculated using SNPAlyze software, Version 7.0 Pro. Power was calculated using a prevalence rate below 0.01 with an additive or a multiplicative model, assuming various degrees of allelic frequencies and the odds ratios for the SNPs.

The potential differences in clinical characteristics (three genotyped patient groups for each SNP) were analyzed using Kruskal-Wallis tests. All statistical analyses were performed using SPSS Statistics software, Version 21 (IBM, Chicago, IL, USA). Following the Kruskal-Wallis tests, post hoc analysis was conducted using two-tailed MannWhitney U-tests. The significance of the $\mathrm{p}^{-v}$ alue for these analyses was set on the basis of the Bonferroni correction (the probability level of $\mathrm{p}<0.05 / 3$ SNPs $=0.0167$ in each analysis) .

The multiple linear regression analysis included the factors that potentially contribute to significantly different clinical characteristics among the genotypes, which were selected on the basis of their significant correlation with altered clinical characteristics by single correlation analysis and were set as independent variables. Finally, stepwise multiple regression analyses were performed for the potential significantly different clinical characteristics among the genotypes as dependent variables, using genotypes as a dummy variable $(0 / 1 ;$ e.g., $\mathrm{G} / \mathrm{G}=1$, $A / G$ and $A / A=0$ ) and the factors showing significant correlation with altered clinical characteristics as independent variables.

\section{Results}

\section{Genetic case-control analyses}

Three SNPs were genotyped in 640 patients with schizophrenia and 640 controls with a genotyping completeness ranging from $99.0 \%$ to $99.6 \%$. Results of the power analyses demonstrated that the power ranged from 9\% (rs2245901) to 17\% (rs6739979). No deviations from the HWE were observed in either the patient or the control samples (all $\mathrm{p}>0.05$, Table-1). None of the SNPs showed any significant 
Table-1 Distribution and statistical analysis of three educational attainment SNPs in Japanese patients with schizophrenia

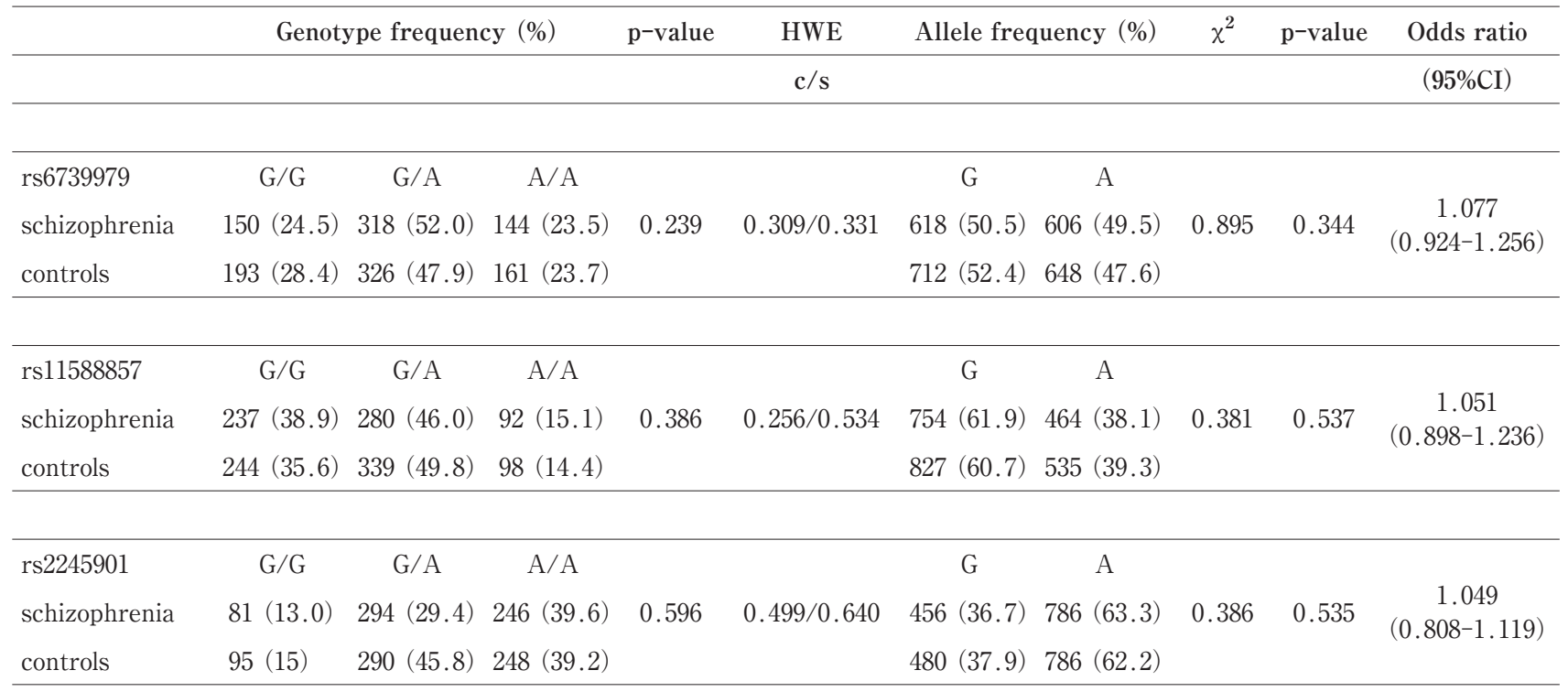

HWE; Hardy-Weinberg equilibrium, CI; confidence interval, c/s; controls/schizophrenia

association between their allelic or genotypic frequencies and schizophrenia. Because rs6739979 and rs2245901 are on the $2 \mathrm{q} 32.3$ region, the LD was calculated between these two SNPs. There was no strong LD between the two SNPs from the total subjects (patients and controls, D'/r'; 0.50/0.14; Figure-1), or from patients $\left(D^{\prime} / r^{2} ; 0.54 / 0.12\right)$ and controls $\left(D^{\prime} / r^{2} ; 0.46 / 0.16\right)$, respectively.

\section{Genotype effect on clinical characteristics}

Among the 640 patients with schizophrenia, 243 patients were admitted to the Juntendo Koshigaya Hospital (Saitama) or Juntendo Hospital (Tokyo) because of acute symptom exacerbation, and their detailed clinical information and symptoms were assessed by a psychiatrist based on detailed interviews with the patients and their family members. These in-patients were also examined using a battery of cognitive assessment tests administered by an expert psychologist. Not all 243 genotyped in-patients could be examined with all cognitive assessment tests depending on their psychiatric conditions (e.g., although BPRS scores could be estimated for all 243 patients, some patients were difficult to examine using complicated assessments, such as WAIS-R). In addition, some clinical information was difficult to assess based on the information available from patients and their family members (e.g., Educational Achievement of Parents of CASH was difficult to assess if the patient's parents are deceased). Thus, the numbers of patients in each clinical variable were different, and detailed case numbers for each battery are shown in Table- 2 .

\section{1) Social status}

Among the eight subscales of CASH, only the "Educational Achievement of Parents" score showed statistically significant differences correlated with the rs11588857 genotype $\left(\chi^{2}=9.07, \mathrm{p}=\right.$ 0.011 ), and individuals with the $\mathrm{A} / \mathrm{G}$ genotype also showed significantly longer "Educational Achievement of Parents" than those with the G/G genotype $\left(\chi^{2}=2.81, \mathrm{p}=0.005\right)$ (Table-2). No significant differences were found correlating with the other subscales of CASH among the rs11588857 genotype, and none of the subscales showed significant correlations with the rs2245901 and rs6739979 genotypes. MPAS, total BPRS scores, and frontal cognitive function tests (verbal fluency test and Stroop test) did not show significantly different results among the genotypes of each of the three SNPs (Table-2).

\section{2) Psychometrics}

The SNP rs2245901 genotype showed a significant correlation with performance IQ (PIQ) levels only $\left(\chi^{2}=7.52, \mathrm{p}=0.023\right)$, and post hoc tests showed that the PIQ levels of individuals with the $G / G$ genotype (G; minor allele) were significantly lower 
Table-2 Clinical characteristics and test scores in each genotype from study participants

\begin{tabular}{|c|c|c|c|c|c|c|c|c|c|c|}
\hline \multirow[t]{2}{*}{ Variables } & \multirow[t]{2}{*}{ Patients } & \multicolumn{3}{|c|}{ rs6739979 } & \multicolumn{3}{|c|}{ rs 11588857} & \multicolumn{3}{|c|}{ rs2245901 } \\
\hline & & $\mathrm{A} / \mathrm{A}$ & $\mathrm{A} / \mathrm{G}$ & $\mathrm{G} / \mathrm{G}$ & $\mathrm{A} / \mathrm{A}$ & $\mathrm{A} / \mathrm{G}$ & $\mathrm{G} / \mathrm{G}$ & $\mathrm{A} / \mathrm{A}$ & $\mathrm{A} / \mathrm{G}$ & $\mathrm{G} / \mathrm{G}$ \\
\hline & ${ }^{*} \mathrm{n}=243$ & $\mathrm{n}=69$ & $\mathrm{n}=108$ & $\mathrm{n}=66$ & $\mathrm{n}=31$ & $\mathrm{n}=122$ & $\mathrm{n}=190$ & $\mathrm{n}=102$ & $\mathrm{n}=109$ & $\mathrm{n}=32$ \\
\hline $\begin{array}{l}\text { Clinical } \\
\text { Variables }\end{array}$ & & \multicolumn{9}{|c|}{ Mean \pm SD $(\min -\max )$} \\
\hline Sex, M/F & & \multicolumn{9}{|c|}{$127 / 137$} \\
\hline $\begin{array}{l}\text { Age, mean } \\
\pm \mathrm{SD}(\mathrm{y})\end{array}$ & & \multicolumn{9}{|c|}{$36.5 \pm 13.2(14-76)$} \\
\hline Onset (y) & & \multicolumn{9}{|c|}{$24.2 \pm 8.6(11-54)$} \\
\hline $\begin{array}{l}\text { Duration of } \\
\text { illness }(\mathrm{y})\end{array}$ & & \multicolumn{9}{|c|}{$12.8 \pm 10.0(0.1-48)$} \\
\hline DUP (m) & & \multicolumn{9}{|c|}{$16.5 \pm 22.0(0.1-96)$} \\
\hline $\begin{array}{l}\text { CED } \\
(\mathrm{mg} / \text { day })\end{array}$ & & \multicolumn{9}{|c|}{$1,037.1 \pm 618.8(2.7-4,562)$} \\
\hline \multicolumn{11}{|l|}{$\begin{array}{l}\text { Clinical } \\
\text { symptoms }\end{array}$} \\
\hline \multirow[t]{2}{*}{$\begin{array}{l}\text { BPRS scores } \\
\text { (total) }\end{array}$} & ${ }^{*} \mathrm{n}=243$ & $\mathrm{n}=69$ & $\mathrm{n}=108$ & $\mathrm{n}=66$ & $\mathrm{n}=31$ & $\mathrm{n}=122$ & $\mathrm{n}=190$ & $\mathrm{n}=102$ & $\mathrm{n}=109$ & $\mathrm{n}=32$ \\
\hline & & $\begin{array}{c}33.3 \pm 11.0 \\
(0-57)\end{array}$ & $\begin{array}{c}36.9 \pm 10.5 \\
(10-82)\end{array}$ & $\begin{array}{c}35.5 \pm 14.5 \\
(0-84)\end{array}$ & $\begin{array}{c}36.0 \pm 9.4 \\
(10-52)\end{array}$ & $\begin{array}{c}35.7 \pm 12.0 \\
(0-82)\end{array}$ & $\begin{array}{c}36.0 \pm 12.4 \\
(0-84)\end{array}$ & $\begin{array}{c}35.4 \pm 11.7 \\
(0-84)\end{array}$ & $\begin{array}{c}35.8 \pm 10.3 \\
(0-60)\end{array}$ & $\begin{array}{c}32.6 \pm 14.9 \\
(0-63)\end{array}$ \\
\hline \multicolumn{11}{|l|}{$\begin{array}{l}\text { Social } \\
\text { status }\end{array}$} \\
\hline $\mathrm{CASH}$ & ${ }^{*} \mathrm{n}=231$ & $\mathrm{n}=61$ & $\mathrm{n}=102$ & $\mathrm{n}=63$ & $\mathrm{n}=29$ & $\mathrm{n}=115$ & $\mathrm{n}=87$ & $\mathrm{n}=93$ & $\mathrm{n}=101$ & $\mathrm{n}=27$ \\
\hline $\begin{array}{l}\text { Marital } \\
\text { Status }\end{array}$ & & $\begin{array}{c}1.4 \pm 0.9 \\
(0-4)\end{array}$ & $\begin{array}{c}1.5 \pm 1.3 \\
(0-9)\end{array}$ & $\begin{array}{c}1.3 \pm 0.8 \\
\quad(1-4)\end{array}$ & $\begin{array}{c}1.5 \pm 1.1 \\
(0-5)\end{array}$ & $\begin{array}{c}1.5 \pm 1.1 \\
(0-9)\end{array}$ & $\begin{array}{c}1.5 \pm 1.1 \\
\quad(1-6)\end{array}$ & $\begin{array}{c}1.4 \pm 0.8 \\
(0-4)\end{array}$ & $\begin{array}{c}1.5 \pm 1.1 \\
(0-6)\end{array}$ & $\begin{array}{c}1.5 \pm 0.9 \\
(1-4)\end{array}$ \\
\hline $\begin{array}{l}\text { Current } \\
\text { Occupation }\end{array}$ & & $\begin{array}{c}8.5 \pm 0.9 \\
(3-9)\end{array}$ & $\begin{array}{c}8.4 \pm 1.1 \\
(3-9)\end{array}$ & $\begin{array}{c}8.5 \pm 1.0 \\
(4-9)\end{array}$ & $\begin{array}{c}8.6 \pm 0.6 \\
(7-9)\end{array}$ & $\begin{array}{c}8.4 \pm 1.0 \\
(3-9)\end{array}$ & $\begin{array}{c}8.5 \pm 1.1 \\
\quad(3-9)\end{array}$ & $\begin{array}{c}8.4 \pm 1.1 \\
(3-9)\end{array}$ & $\begin{array}{c}8.5 \pm 1.0 \\
(3-9)\end{array}$ & $\begin{array}{c}8.5 \pm 0.8 \\
(7-9)\end{array}$ \\
\hline $\begin{array}{l}\text { Previous } \\
\text { Occupation }\end{array}$ & & $\begin{array}{c}6.6 \pm 2.0 \\
(2-9)\end{array}$ & $\begin{array}{c}6.7 \pm 1.8 \\
(2-9)\end{array}$ & $\begin{array}{c}7.0 \pm 1.8 \\
(2-9)\end{array}$ & $\begin{array}{c}6.4 \pm 1.9 \\
(2-9)\end{array}$ & $\begin{array}{c}6.6 \pm 1.9 \\
(3-9)\end{array}$ & $\begin{array}{c}6.8 \pm 1.8 \\
(2-9)\end{array}$ & $\begin{array}{c}6.6 \pm 1.8 \\
(2-9)\end{array}$ & $\begin{array}{c}6.7 \pm 1.9 \\
(2-9)\end{array}$ & $\begin{array}{c}6.6 \pm 1.8 \\
(2-9)\end{array}$ \\
\hline $\begin{array}{l}\text { Occupation } \\
\text { of Parents }\end{array}$ & & $\begin{array}{c}4.3 \pm 2.1 \\
\quad(1-9)\end{array}$ & $\begin{array}{c}4.2 \pm 1.7 \\
(1-9)\end{array}$ & $\begin{array}{c}4.4 \pm 1.7 \\
(0-9)\end{array}$ & $\begin{array}{c}4.0 \pm 2.3 \\
(1-8)\end{array}$ & $\begin{array}{c}4.6 \pm 1.8 \\
(1-9)\end{array}$ & $\begin{array}{c}4.0 \pm 1.5 \\
(0-9)\end{array}$ & $\begin{array}{c}4.6 \pm 1.9 \\
(1-9)\end{array}$ & $\begin{array}{c}4.2 \pm 1.7 \\
(1-9)\end{array}$ & $\begin{array}{c}3.9 \pm 1.7 \\
(0-7)\end{array}$ \\
\hline $\begin{array}{l}\text { Educational } \\
\text { Achievement } \\
\text { of Subject }\end{array}$ & & $\begin{array}{l}12.6 \pm 2.1 \\
\quad(9-16)\end{array}$ & $\begin{array}{l}12.4 \pm 2.4 \\
\quad(9-20)\end{array}$ & $\begin{array}{l}12.6 \pm 2.7 \\
\quad(8-21)\end{array}$ & $\begin{array}{l}12.6 \pm 2.2 \\
\quad(9-16)\end{array}$ & $\begin{array}{l}12.5 \pm 2.4 \\
\quad(9-21)\end{array}$ & $\begin{array}{l}12.2 \pm 2.5 \\
\quad(8-18)\end{array}$ & $\begin{array}{l}12.3 \pm 2.2 \\
\quad(9-16)\end{array}$ & $\begin{array}{l}12.6 \pm 2.5 \\
\quad(9-21)\end{array}$ & $\begin{array}{l}12.0 \pm 2.6 \\
\quad(8-16)\end{array}$ \\
\hline $\begin{array}{l}\text { Educational } \\
\text { Performance }\end{array}$ & & $\begin{array}{l}3.0 \pm 0.8 \\
(1-5)\end{array}$ & $\begin{array}{l}3.0 \pm 0.9 \\
(1-5)\end{array}$ & $\begin{array}{l}3.0 \pm 1.2 \\
\quad(1-7)\end{array}$ & $\begin{array}{l}2.9 \pm 0.8 \\
\quad(2-4)\end{array}$ & $\begin{array}{l}3.0 \pm 1.1 \\
\quad(1-7)\end{array}$ & $\begin{array}{l}3.0 \pm 0.8 \\
(1-4)\end{array}$ & $\begin{array}{l}3.0 \pm 0.9 \\
(1-5)\end{array}$ & $\begin{array}{l}3.0 \pm 0.9 \\
(1-7)\end{array}$ & $\begin{array}{l}2.7 \pm 1.1 \\
\quad(1-6)\end{array}$ \\
\hline \multirow{4}{*}{\multicolumn{2}{|c|}{$\begin{array}{l}\text { Educational } \\
\text { Achievement } \\
\text { of Parents }\end{array}$}} & $\begin{array}{l}12.7 \pm 3.3 \\
\quad(9-21)\end{array}$ & $\begin{array}{l}12.0 \pm 3.7 \\
\quad(2-21)\end{array}$ & $\begin{array}{l}12.4 \pm 3.2 \\
\quad(6-16)\end{array}$ & $\begin{array}{l}13.3 \pm 3.7^{* *} \\
\quad(9-21)\end{array}$ & $\begin{array}{l}12.8 \pm 2.9^{* *} \\
\quad(9-21)\end{array}$ & $\begin{array}{l}10.5 \pm 3.8^{* *} \\
\quad(2-16)\end{array}$ & $\begin{array}{l}12.0 \pm 3.4 \\
\quad(2-21)\end{array}$ & $\begin{array}{l}12.2 \pm 3.7 \\
\quad(2-21)\end{array}$ & $\begin{array}{l}12.5 \pm 3.7 \\
\quad(6-16)\end{array}$ \\
\hline & & \multirow{3}{*}{\multicolumn{3}{|c|}{$\begin{array}{c}\text { Post hoc analysis } \\
\text { (Mann-Whitney U-tests) }\end{array}$}} & $\mathrm{A} / \mathrm{A}$ vs $\mathrm{G}$ & $/ G ; \chi^{2}=2.04$, & $\mathrm{p}=0.042$ & & & \\
\hline & & & & & $\mathrm{A} / \mathrm{G}$ vs $\mathrm{G} /$ & $G ; \chi^{2}=2.81$, & $p=0.005$ & & & \\
\hline & & & & & $\mathrm{A} / \mathrm{A}$ vs $\mathrm{A}$ & $/ G ; x^{2}=0.36$ & $\mathrm{p}=0.717$ & & & \\
\hline \multirow[t]{3}{*}{$\begin{array}{l}\text { Living Cir- } \\
\text { cumstances }\end{array}$} & & $\begin{array}{c}2.3 \pm 1.0 \\
(1-7)\end{array}$ & $\begin{array}{c}2.4 \pm 0.9 \\
(1-7)\end{array}$ & $\begin{array}{c}2.4 \pm 0.9 \\
(1-5)\end{array}$ & $\begin{array}{c}2.4 \pm 0.9 \\
(2-6)\end{array}$ & $\begin{array}{c}2.4 \pm 1.0 \\
(1-7)\end{array}$ & $\begin{array}{c}2.4 \pm 0.9 \\
(1-6)\end{array}$ & $\begin{array}{c}2.3 \pm 0.8 \\
(1-6)\end{array}$ & $\begin{array}{c}2.4 \pm 1.1 \\
(1-7)\end{array}$ & $\begin{array}{c}2.3 \pm 0.7 \\
(2-4)\end{array}$ \\
\hline & & \multicolumn{3}{|c|}{ rs6739979 } & \multicolumn{3}{|c|}{ rs11588857 } & \multicolumn{3}{|c|}{ rs2245901 } \\
\hline & & $\mathrm{A} / \mathrm{A}$ & $\mathrm{A} / \mathrm{G}$ & $\mathrm{G} / \mathrm{G}$ & $\mathrm{A} / \mathrm{A}$ & $\mathrm{A} / \mathrm{G}$ & $\mathrm{G} / \mathrm{G}$ & $\mathrm{A} / \mathrm{A}$ & $\mathrm{A} / \mathrm{G}$ & $\mathrm{G} / \mathrm{G}$ \\
\hline MPAS & ${ }^{*} \mathrm{n}=168$ & $\mathrm{n}=46$ & $\mathrm{n}=69$ & $\mathrm{n}=45$ & $\mathrm{n}=21$ & $\mathrm{n}=84$ & $\mathrm{n}=56$ & $\mathrm{n}=74$ & $\mathrm{n}=77$ & $\mathrm{n}=17$ \\
\hline $\begin{array}{l}\text { Withdrawal } \\
(6-12 \mathrm{y})\end{array}$ & & $\begin{array}{l}1.0 \pm 0.9 \\
(0-3)\end{array}$ & $\begin{array}{l}1.0 \pm 0.9 \\
(0-3)\end{array}$ & $\begin{array}{l}0.8 \pm 0.8 \\
\quad(0-3)\end{array}$ & $\begin{array}{l}0.9 \pm 0.8 \\
\quad(0-3)\end{array}$ & $\begin{array}{l}0.9 \pm 0.9 \\
(0-3)\end{array}$ & $\begin{array}{l}1.0 \pm 0.9 \\
(0-3)\end{array}$ & $\begin{array}{l}1.0 \pm 0.8 \\
(0-3)\end{array}$ & $\begin{array}{c}0.9 \pm 0.9 \\
(0-3)\end{array}$ & $\begin{array}{c}0.7 \pm 0.7 \\
(0-2)\end{array}$ \\
\hline $\begin{array}{l}\text { Peer } \\
\text { Relationship } \\
(6-12 y)\end{array}$ & & $\begin{array}{c}1.2 \pm 1.0 \\
\quad(0-3)\end{array}$ & $\begin{array}{c}0.9 \pm 0.9 \\
\quad(0-3)\end{array}$ & $\begin{array}{c}1.0 \pm 1.0 \\
(0-3)\end{array}$ & $\begin{array}{c}1.1 \pm 0.9 \\
(0-3)\end{array}$ & $\begin{array}{c}1.0 \pm 0.9 \\
(0-3)\end{array}$ & $\begin{array}{c}1.0 \pm 0.9 \\
(0-3)\end{array}$ & $\begin{array}{c}1.0 \pm 0.9 \\
(0-3)\end{array}$ & $\begin{array}{c}1.0 \pm 1.0 \\
(0-3)\end{array}$ & $\begin{array}{c}1.0 \pm 0.8 \\
(0-2)\end{array}$ \\
\hline
\end{tabular}




\begin{tabular}{|c|c|c|c|c|c|c|c|c|c|}
\hline $\begin{array}{l}\text { Interests } \\
(6-12 \mathrm{y})\end{array}$ & $\begin{array}{l}1.2 \pm 0.8 \\
\quad(0-3)\end{array}$ & $\begin{array}{l}1.1 \pm 0.8 \\
(0-3)\end{array}$ & $\begin{array}{l}0.9 \pm 0.9 \\
(0-3)\end{array}$ & $\begin{array}{l}1.2 \pm 0.8 \\
(0-3)\end{array}$ & $\begin{array}{l}1.0 \pm 0.8 \\
\quad(0-3)\end{array}$ & $\begin{array}{c}1.0 \pm 0.8 \\
(0-3)\end{array}$ & $\begin{array}{l}1.1 \pm 0.7 \\
(0-3)\end{array}$ & $\begin{array}{l}1.1 \pm 0.9 \\
(0-3)\end{array}$ & $\begin{array}{l}0.7 \pm 0.7 \\
\quad(0-2)\end{array}$ \\
\hline $\begin{array}{l}\text { Withdrawal } \\
(13-21 y)\end{array}$ & $\begin{array}{c}1.8 \pm 1.9 \\
(0-12)\end{array}$ & $\begin{array}{c}1.4 \pm 1.2 \\
\quad(0-6)\end{array}$ & $\begin{array}{c}1.2 \pm 0.9 \\
(0-3)\end{array}$ & $\begin{array}{c}1.3 \pm 0.9 \\
(0-3)\end{array}$ & $\begin{array}{c}1.4 \pm 1.6 \\
(0-12)\end{array}$ & $\begin{array}{c}1.5 \pm 1.2 \\
\quad(0-6)\end{array}$ & $\begin{array}{c}1.4 \pm 1.1 \\
(0-6)\end{array}$ & $\begin{array}{c}1.51 \pm 1.5 \\
(0-12)\end{array}$ & $\begin{array}{c}1.24 \pm 0.8 \\
\quad(0-3)\end{array}$ \\
\hline $\begin{array}{l}\text { Peer } \\
\text { Relationship } \\
(13-21 \mathrm{y})\end{array}$ & $\begin{array}{c}1.5 \pm 0.9 \\
(0-3)\end{array}$ & $\begin{array}{c}1.2 \pm 0.8 \\
\quad(0-3)\end{array}$ & $\begin{array}{c}1.4 \pm 1.0 \\
(0-3)\end{array}$ & $\begin{array}{c}1.4 \pm 0.9 \\
(0-3)\end{array}$ & $\begin{array}{c}1.2 \pm 0.9 \\
\quad(0-3)\end{array}$ & $\begin{array}{c}1.4 \pm 0.9 \\
(0-3)\end{array}$ & $\begin{array}{c}1.3 \pm 0.9 \\
(0-3)\end{array}$ & $\begin{array}{c}1.4 \pm 1.0 \\
(0-3)\end{array}$ & $\begin{array}{c}1.4 \pm 0.9 \\
(0-3)\end{array}$ \\
\hline $\begin{array}{l}\text { Interests } \\
(13-21 y)\end{array}$ & $\begin{array}{l}1.5 \pm 0.9 \\
\quad(0-3)\end{array}$ & $\begin{array}{l}1.4 \pm 0.8 \\
\quad(0-3)\end{array}$ & $\begin{array}{c}1.2 \pm 1.0 \\
(0-3)\end{array}$ & $\begin{array}{l}1.5 \pm 0.9 \\
(0-3)\end{array}$ & $\begin{array}{l}1.3 \pm 0.9 \\
(0-3)\end{array}$ & $\begin{array}{l}1.4 \pm 0.9 \\
(0-3)\end{array}$ & $\begin{array}{l}1.4 \pm 0.8 \\
(0-3)\end{array}$ & $\begin{array}{l}1.3 \pm 1.3 \\
(0-3)\end{array}$ & $\begin{array}{l}1.4 \pm 0.9 \\
(0-2)\end{array}$ \\
\hline $\begin{array}{l}\text { Socio-sexual } \\
\text { adjustment } \\
(13-21 y)\end{array}$ & $\begin{array}{c}1.7 \pm 0.9 \\
(0-3)\end{array}$ & $\begin{array}{c}1.6 \pm 0.8 \\
(0-3)\end{array}$ & $\begin{array}{c}1.8 \pm 1.0 \\
(0-3)\end{array}$ & $\begin{array}{c}1.6 \pm 0.7 \\
(0-3)\end{array}$ & $\begin{array}{c}1.6 \pm 0.8 \\
(0-3)\end{array}$ & $\begin{array}{c}1.6 \pm 1.0 \\
(0-3)\end{array}$ & $\begin{array}{c}1.7 \pm 0.9 \\
(0-3)\end{array}$ & $\begin{array}{c}1.6 \pm 0.8 \\
(0-3)\end{array}$ & $\begin{array}{c}1.5 \pm 0.9 \\
(0-3)\end{array}$ \\
\hline Psychometrics & & & & & & & & & \\
\hline $\begin{array}{l}\text { Frontal } \\
\text { lobe } \\
\text { function }\end{array}$ & $\mathrm{n}=33$ & $\mathrm{n}=62$ & $\mathrm{n}=32$ & $\mathrm{n}=13$ & $\mathrm{n}=65$ & $\mathrm{n}=47$ & $\mathrm{n}=57$ & $\mathrm{n}=58$ & $\mathrm{n}=15$ \\
\hline $\begin{array}{l}\text { Verbal } \\
\text { fluency test }\end{array}$ & $\begin{array}{c}24.2 \pm 10.5 \\
\quad(5-50)\end{array}$ & $\begin{array}{c}26.5 \pm 11.4 \\
\quad(7-67)\end{array}$ & $\begin{array}{c}22.4 \pm 10.3 \\
\quad(6-49)\end{array}$ & $\begin{array}{l}27.9 \pm 15.0 \\
\quad(8-67)\end{array}$ & $\begin{array}{c}25.2 \pm 10.9 \\
\quad(7-54)\end{array}$ & $\begin{array}{c}23.8 \pm 9.2 \\
\quad(6-42)\end{array}$ & $\begin{array}{c}24.1 \pm 9.5 \\
\quad(7-50)\end{array}$ & $\begin{array}{c}25.5 \pm 11.6 \\
(5-67)\end{array}$ & $\begin{array}{c}25.6 \pm 12.3 \\
\quad(6-45)\end{array}$ \\
\hline $\begin{array}{l}\text { Stroop test } \\
\text { (time) }\end{array}$ & $\begin{array}{c}100.9 \pm 46.4 \\
(24-271)\end{array}$ & $\begin{array}{c}97.9 \pm 56.3 \\
(10-335)\end{array}$ & $\begin{array}{c}116.8 \pm 62.9 \\
(-6-231)\end{array}$ & $\begin{array}{c}110.8 \pm 52.3 \\
\quad(30-220)\end{array}$ & $\begin{array}{c}96.3 \pm 56.7 \\
(-6-335)\end{array}$ & $\begin{array}{c}115.6 \pm 53.4 \\
(24-231)\end{array}$ & $\begin{array}{c}104.4 \pm 59.9 \\
(10-335)\end{array}$ & $\begin{array}{c}101.4 \pm 53.4 \\
\quad(-6-271)\end{array}$ & $\begin{array}{c}122.1 \pm 53.3 \\
\quad(37-220) \\
\end{array}$ \\
\hline $\begin{array}{ll}\text { Intelligence } & { }^{*} \mathrm{n}=64 \\
\text { scales } & \mathrm{n}\end{array}$ & $\mathrm{n}=18$ & $\mathrm{n}=28$ & $\mathrm{n}=18$ & $\mathrm{n}=10$ & $\mathrm{n}=29$ & $\mathrm{n}=25$ & $\mathrm{n}=26$ & $\mathrm{n}=29$ & $\mathrm{n}=9$ \\
\hline JART 100 & $\begin{array}{c}78.9 \pm 16.5 \\
(55-110)\end{array}$ & $\begin{array}{c}81.5 \pm 18.0 \\
(55-122)\end{array}$ & $\begin{array}{c}80.9 \pm 17.9 \\
(57-114)\end{array}$ & $\begin{array}{c}86.6 \pm 18.1 \\
(66-110)\end{array}$ & $\begin{array}{c}84.5 \pm 18.6 \\
(55-122)\end{array}$ & $\begin{array}{c}76.8 \pm 17.3 \\
(57-114)\end{array}$ & $\begin{array}{c}82.9 \pm 17.2 \\
(60-114)\end{array}$ & $\begin{array}{c}83.6 \pm 16.9 \\
(55-114)\end{array}$ & $\begin{array}{c}74.7 \pm 22.9 \\
(57-122)\end{array}$ \\
\hline $\begin{array}{l}\text { WAIS-R } \\
\text { (FSIQ) }\end{array}$ & $\begin{array}{c}73.1 \pm 14.8 \\
(48-114)\end{array}$ & $\begin{array}{c}74.7 \pm 13.7 \\
(52-101)\end{array}$ & $\begin{array}{c}76.3 \pm 18.9 \\
(44-110)\end{array}$ & $\begin{array}{c}79.8 \pm 18.5 \\
(64-114)\end{array}$ & $\begin{array}{c}76.0 \pm 13.9 \\
(48-110)\end{array}$ & $\begin{array}{c}70.6 \pm 15.1 \\
(44-110)\end{array}$ & $\begin{array}{c}75.0 \pm 15.5 \\
(44-114)\end{array}$ & $\begin{array}{c}77.0 \pm 16.0 \\
(48-110)\end{array}$ & $\begin{array}{c}70.2 \pm 7.6 \\
\quad(57-80)\end{array}$ \\
\hline (VIQ) & $\begin{array}{c}77.6 \pm 15.0 \\
(55-119)\end{array}$ & $\begin{array}{c}79.0 \pm 15.6 \\
(48-111)\end{array}$ & $\begin{array}{c}84.5 \pm 18.9 \\
(55-121)\end{array}$ & $\begin{array}{c}83.4 \pm 18.5 \\
(60-119)\end{array}$ & $\begin{array}{c}81.8 \pm 15.0 \\
(48-113)\end{array}$ & $\begin{array}{c}75.8 \pm 15.5 \\
(53-119)\end{array}$ & $\begin{array}{c}81.2 \pm 17.3 \\
(53-121)\end{array}$ & $\begin{array}{c}82.1 \pm 15.6 \\
(55-119)\end{array}$ & $\begin{array}{c}75.6 \pm 14.7 \\
(48-97)\end{array}$ \\
\hline (PIQ) & $\begin{array}{c}73.9 \pm 15.5 \\
(49-103)\end{array}$ & $\begin{array}{c}70.9 \pm 16.0 \\
(29-97)\end{array}$ & $\begin{array}{c}74.5 \pm 16.7 \\
(54-103)\end{array}$ & $\begin{array}{c}78.8 \pm 18.6 \\
(56-103)\end{array}$ & $\begin{array}{c}71.2 \pm 16.4 \\
(29-103)\end{array}$ & $\begin{array}{c}71.7 \pm 13.6 \\
(54-97) \\
\end{array}$ & $\begin{array}{l}75.7 \pm 13.6^{* *} \\
(54-103) \\
\end{array}$ & $\begin{array}{c}* * 74.5 \pm 16.0^{*} \\
(49-103)\end{array}$ & $\begin{array}{c}59.8 \pm 12.4^{* *} \\
(29-71) \\
\end{array}$ \\
\hline & & & & \multicolumn{3}{|c|}{$\begin{array}{c}\text { Post hoc analysis } \\
\text { (Mann-Whitney U-tests) }\end{array}$} & \multicolumn{3}{|c|}{$\begin{array}{c}\mathrm{A} / \mathrm{A} \text { vs } \mathrm{G} / \mathrm{G} ; \chi^{2}=-2.83, \mathrm{p}=0.004 \\
\mathrm{~A} / \mathrm{G} \text { vs } \mathrm{G} / \mathrm{G} ; \chi^{2}=-2.20, \mathrm{p}=0.027 \\
\mathrm{~A} / \mathrm{A} \text { vs } \mathrm{A} / \mathrm{G} ; \chi^{2}=-0.52, \mathrm{p}=0.604\end{array}$} \\
\hline
\end{tabular}

BPRS; Brief Psychiatric Rating Scale, CASH; The Comprehensive Assessment of Symptoms and History, CED; Chlorpromazine-equivalent dose, DUP; Duration of Untreated Psychosis, JART; Japanese version of the National Adult Reading Test, FSIQ; full score intelligence quotient, VIQ; verbal IQ, PIQ; performance IQ, MPAS; Modified; Premorbid Adjustment Scale.

${ }^{*}$ Because some patients were difficult to be examined complicated assessments, such as WAIS-R, and some correct clinical information was difficult to know from patients and their family (e.g. Educational Achievement of Parents of CASH, because of death of parents). Thus, the numbers of patients in each clinical variable were different.

${ }^{* *} \mathrm{p}^{-}$-values with statistical significance among the genotypes are in bold, then post hoc analysis were performed between two genotype combinations.

than those with the A/A genotype $\left(\chi^{2}=-2.83, \mathrm{p}=\right.$ 0.004) with Bonferroni correction. Individuals with the $\mathrm{A} / \mathrm{G}$ genotype also showed lower PIQ levels than the A/A genotype, but these levels were not statistically significant by a small margin when the Bonferroni correction for multiple comparisons was considered $\left(\chi^{2}=-2.20, \mathrm{p}=0.027\right)$. Full score IQ (FSIQ) and verbal IQ (VIQ) did not show any significant differences between the three genotypes (Table-2). JART scores estimating premorbid intelligence did not show alterations based on genotype for all SNPs.
3) Multiple linear regression analysis

To find the confounding factors that could affect the aforementioned significant findings (PIQ and "Educational Achievement of Parents"), single correlation analyses were performed by using all obtained clinical variables and assessment scores listed in Table-2. PIQ showed significant negative correlations with total BPRS scores $(r=-0.365$, $\mathrm{p}=0.002)$ and daily dose of antipsychotics ( $\mathrm{r}=$ $-0.291, \mathrm{p}=0.017$ ), whereas "Educational Achievement of Parents" showed a significant positive correlation with "Educational Achievement of 
Subject" $\quad(\mathrm{r}=0.328, \mathrm{p}=0.001)$ and negative correlation with duration of illness $(r=-0.302, p=0.002)$ and age at admission $(\mathrm{r}=-0.368, \mathrm{p}<0.001)$.

The stepwise multiple linear regression analysis was performed using PIQ level as a dependent variable and total BPRS scores and daily dose of antipsychotics as potential independent variables for the rs2245901 genotype. For the rs 11588857 genotype, this analysis was performed with "Educational Achievement of Parents" as a dependent variable and "Educational Achievement of Subject," duration of illness, and age at admission as potential independent variables. Genotypes were included as dummy variables $(0 / 1)$.

The analysis for PIQ showed that the rs2245901 genotype and the total BPRS scores were both significant in predicting the PIQ levels in the first stage of the analysis $(p=0.007$ and $p=0.012$, respectively), whereas the daily dose of antipsychotics was not (Table-2). The predictive equation for PIQ levels was then as follows: PIQ level $=89.2+-0.317$ $\times$ (rs2245901 genotype; homozygous minor allele $\mathrm{G} / \mathrm{G}=1$, genotypes $\mathrm{AA}$ and $\mathrm{A} / \mathrm{G}=0)+-0.291 \times($ total BPRS scores). The analysis for the "Educational Achievement of Parents" showed that the rs11588857 genotype, "Educational Achievement of Subject" and duration of illness were excluded in the first stage of the analysis in predicting the "Educational Achievement of Parents," whereas only the age at admission was significant (Table-2). The predictive equation for "Educational Achievement of Parents" was then as follows: "Educational Achievement of Parents (years)" $=16.3+-0.414 \times$ (years of age at admission).

\section{Discussion}

The present study investigated whether three SNPs--rs6739979, rs11588857, and rs2245901--which have previously been implicated in GWASs of educational attainment, could also be associated with the onset and/or education-related clinical features of Japanese patients with schizophrenia.

Results of the present study indicate that these SNPs are not genetic risk factors for the onset of schizophrenia in Japanese patients. These results are consistent with previous results because the genetic regions where these three SNPs are located have not been identified as genetic risk factors for schizophrenia in most previous GWASs (GWAS catalog: https://www.ebi.ac.uk/gwas/home), with the exception of earlier studies examining primarily individuals with European ancestry using earlier methods (low density of probes) for the 2q32.3 region (for rs6739979 and rs2245901) (Figure-1) ${ }^{24)-27)}$

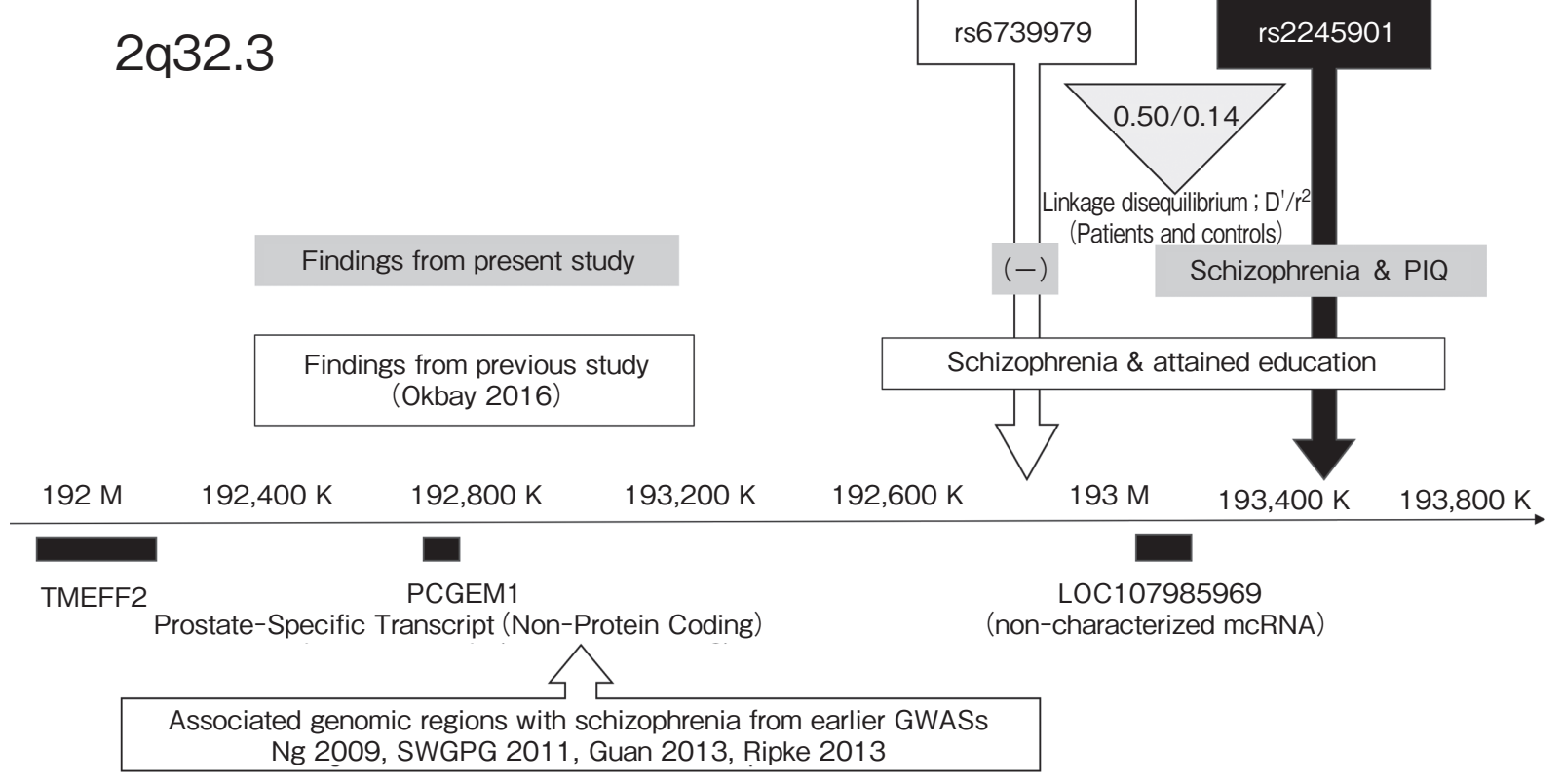

Figure-1 A comprehensive schema for the relative location of SNPs and genes of interest on 2q32.3, and findings of the present and previous investigations

Genomic positions were obtained from the GRCh38.p7 assembly.

SWGPG: Schizophrenia Psychiatric Genome-Wide Association Study 
and the 1q32.1 region (for rs11588857) ${ }^{28)}$. Thus, these regions do not appear to be genetic risk factors for Japanese patients with schizophrenia.

Two of the three SNPs, rs11588857 and rs2245901 investigated in the present study showed some correlation with the clinical features of schizophrenia related to education or cognitive function. For the rs11588857, its genotype showed significant difference for "Educational Achievement of Parents" between individuals with the $A / G$ and G/G genotype, but not between the A/A and G/G genotypes; however, genetically the difference should be more apparent. In addition, this genotype was excluded in the first stage of the stepwise multiple linear regression analysis in predicting the "Educational Achievement of Parents". Based on these results, it was difficult to determine that rs11588857 could affect one of the educational social statuses, "Educational Achievement of Parents."

While rs2245901 showed a significant correlation with the PIQ of schizophrenia patients after multiple linear regression analysis. This SNP failed to show a correlation with epidemiological assessments of education features, such as years of education, although a recent GWAS did report a correlation between this SNP and years of education. However, the significance of this correlation could not survive after the Bonferroni correction was applied, though its correlation with schizophrenia remained significant (see recent published additional sheet "3.2 SNP Look-up" ${ }^{12}$. Of course, there may be discrepancies between the methods used in the recent GWAS and the present study, both in the number and background (diagnosis) of subjects. Although the sample size used in the recent GWAS was large $(\mathrm{N}=218,795)^{12)}$, other important information regarding the patients' detailed clinical features, such as severity of symptoms and social adaptation, were not considered. Our present sample size was smaller $(\mathrm{N}=$ 108), but the heterogeneity of schizophrenia must be considered, and all patients selected for the present study displayed exacerbated symptoms. In addition, we considered not only objective, epidemiological assessments of educational information, but also further detailed clinical information, such as cognitive functions with psychological assessment, severity of symptoms, and daily antipsychotics dose. Interestingly, when patients were limited to
Japanese in-patients with severe symptoms at the time of admission, multiple regression analyses showed that SNP rs2245901 in the 2q32.3 region survived as an independent variable and is correlated with PIQ levels in schizophrenia patients (Table-2). In addition, the PIQ of Japanese patients with schizophrenia markedly declined compared with that of healthy controls, whereas the FSIQ and VIQ of these patients did not ${ }^{29}$. These features were also reported in patients with schizophrenia speaking a non-Japanese language ${ }^{30-32}$. Taken together, these findings suggest that genetic region 2q32.3 may play an important role in the disturbance of cognitive function in schizophrenia.

Assumed premorbid IQ (based on JART scores) from patients with schizophrenia were not affected by rs2245901 and the other two SNPs. Indeed, it has been reported that over $50 \%$ of patients with schizophrenia showed declined intelligence (deteriorated IQ; from premorbid to present IQ) ${ }^{33-36)}$. Our previous study of 446 patients with schizophrenia showed that approximately $30 \%$ of patients had a decline of less than 10 IQ points, whereas approximately $70 \%$ of patients showed a greater deterioration in their IQ, with some displaying a severe decline of 30 points or greater $(13.5 \%)^{3)}$. Thus, the genetic influence of rs2245901 on $2 \mathrm{q} 32.3$ may play a role in the deteriorated IQ of these patients, and not the patients with preserved IQ.

As of this writing, although the use of atypical antipsychotics for cognitive impairments is limited in schizophrenia, several cognitive rehabilitation programs, such as The Neuropsychological Educational Approach to Remediation, should intervene at the early stages of the disease ${ }^{37}$. But considering that some patients dropped out of these programs ${ }^{37)}{ }^{38)}$, evaluating this SNP in schizophrenia patients may be useful for choosing suitable cognitive remediation programs for each patient during early intervention.

The present study had a number of limitations. First, the type of antipsychotics and concomitant medications, such as anti-cholinergics ${ }^{39)}$ and benzodiazepines ${ }^{40)}$, used by schizophrenia patients may reduce their cognitive function. The use of these medications was not considered in the present study, although daily doses of antipsychotics were analyzed and did not affect PIQ. Second, we used the WAIS-R scale for the 
measurement of IQ; however, clinical features of schizophrenia also include impairment of Verbal Comprehension, Working Memory, Perceptual Organization, and Processing Speed, which are measured using the WAIS-III scale, and may be correlated with $\mathrm{PIQ}^{29)}{ }^{41}$. We are accumulating data for these four indices using the WAIS-III scale and will further investigate the relationship between candidate SNPs and these indices. Third, the functions of this SNP, the minor G allele of rs224590, are difficult to elucidate because it is an intergenic SNP, rather than a coding or a regulatory SNP (Figure-1). In addition, the function of the nearest gene, LOC107985969, situated up-stream of rs2245901, was unknown until recently. Furthermore, as there is no strong LD between SNPs rs6739979 and rs2245901 (Figure-1), the relationship between rs2245901 and PIQ does not appear to reflect the genetic features of LOC107985969. Although we could not find any association between the up-stream rs6739979 (another intergenic SNP) and observed educational attainment and cognitive function in schizophrenia, a previous study showed a relationship between this intergenic SNP and attained education ${ }^{12)}$. The 5 ' neighboring gene for rs6739979 is prostate-specific transcript 1 (PCGEM1), which produces a long intergenic nonprotein coding RNA and plays a role in the progression of prostate cancer ${ }^{42}$. Interestingly, recent GWASs have demonstrated that the genetic region of $P C G E M 1$ sometimes shows an association with schizophrenia ${ }^{27)}{ }^{43)}$-45), and another study revealed its association with brain morphology in schizophrenia ${ }^{46)}$. Another gene, Transmembrane Protein With EGF Like And Two Follistatin-Like Domains 2 (TMEFF2) is situated more up-stream (Figure-1), is widely expressed in the brain, especially during hippocampal formation, and could be a survival factor for hippocampal and mesencephalic neurons ${ }^{47}{ }^{48}$. This gene may be involved in the relationship between the current genetic findings and disturbed brain function in schizophrenia. Fourth, we have chosen three candidate SNPs from the recent and largest GWAS showing the relationship between these SNPs and both schizophrenia and educational attainments. However, there were some candidate SNPs in other genetic regions reported in other GWASs examining educational attainments without considering their relationship to schizophrenia ${ }^{49)-51)}$. In particular, a recent GWAS focusing on schizophrenia and educational attainment (education years) showed other candidate SNPs as pleiotropic loci, although nearest SNP (rs1913145, Ch2; 193753869) to those of the present study failed to show a relationship with either schizophrenia or education years ${ }^{52}$. Further investigations are needed to verify the role of these SNPs in cognitive dysfunction in schizophrenia.

In conclusion, the three candidate SNPs investigated during the present study do not appear to be genetic risk factors for schizophrenia in Japanese patients. However, the genetic influence of rs2245901 may be associated with the deteriorated PIQ seen in some schizophrenia patients, and not those patients having preserved IQ. In addition, genetic region $2 \mathrm{q} 32.3$ may genetically influence attained education and decline of cognitive function in patients with schizophrenia.

\section{Acknowledgements}

This work was supported by the Juntendo Institute of Mental Health from 2017 (201701) to 2018 (201801).

\section{References}

1) Owen MJ, Sawa A, Mortensen PB: Schizophrenia. Lancet, 2016; 388: 86-97.

2) Condray R, van Kammen DP, Steinhauer SR, Kasparek A, Yao JK: Language comprehension in schizophrenia: trait or state indicator? Biol Psychiatry, 1995; 38: 287296.

3) Fujino H, Sumiyoshi C, Yasuda Y, et al: Estimated cognitive decline in patients with schizophrenia: A multicenter study. Psychiatry Clin Neurosci, 2017; 71: 294-300.

4) Mahurin RK, Velligan DI, Miller AL: Executive-frontal lobe cognitive dysfunction in schizophrenia: a symptom subtype analysis. Psychiatry Res, 1998; 79: 139-149.

5) Snitz BE, Macdonald AW 3rd, Carter CS: Cognitive deficits in unaffected first-degree relatives of schizophrenia patients: a meta-analytic review of putative endophenotypes. Schizophr Bull, 2006; 32: 179-194.

6) Cohen JD, Servan-Schreiber D: Context, cortex, and dopamine: a connectionist approach to behavior and biology in schizophrenia. Psychol Rev, 1992; 99: 45-77.

7) Javitt DC: Treatment of negative and cognitive symptoms. Curr Psychiatry Rep, 1999; 1: 25-30.

8) Miles AA, Heinrichs RW, Ammari N, et al: Stability and change in symptoms, cognition, and community outcome in schizophrenia. Schizophr Res, 2014; 152: 435-439.

9) Siegrist K, Millier A, Amri I, Aballéa S, Toumi M: Association between social contact frequency and 
negative symptoms, psychosocial functioning and quality of life in patients with schizophrenia. Psychiatry Res, 2015; 230: 860-866.

10) Tamminga CA: The neurobiology of cognition in schizophrenia. J Clin Psychiatry, 2006; 67: e11.

11) Rietveld CA, Medland SE, Derringer J, et al: GWAS of 126,559 individuals identifies genetic variants associated with educational attainment. Science, 2013; 340: 14671471.

12) Okbay A, Beauchamp JP, Fontana MA, et al: Genomewide association study identifies 74 loci associated with educational attainment. Nature, 2016; 533: 539-542.

13) Inada $T$, Inagaki $A$ : Psychotropic dose equivalence in Japan. Psychiatry Clin Neurosci, 2015; 69: 440-447.

14) Katsuta N, Ohnuma T, Maeshima H, et al: Significance of measurements of peripheral carbonyl stress markers in a cross-sectional and longitudinal study in patients with acute-stage schizophrenia. Schizophr Bull, 2014; 40: $1366-1373$.

15) Andreasen NC, Flaum M, Arndt S: The Comprehensive Assessment of Symptoms and History (CASH). An instrument for assessing diagnosis and psychopathology. Arch Gen Psychiatry, 1992; 49: 615-623.

16) Gupta S, Rajaprabhakaran R, Arndt S, Flaum M, Andreasen NC: Premorbid adjustment as a predictor of phenomenological and neurobiological indices in schizophrenia. Schizophr Res, 1995; 16: 189-197.

17) Wechsler D: Wechsler Adult Intelligence ScaleRevised: San Antonio, TX: Psychological Corporation, 1981.

18) Matsuoka K, Uno M, Kasai K, Koyama K, Kim Y: Estimation of premorbid IQ in individuals with Alzheimer's disease using Japanese ideographic script (Kanji) compound words: Japanese version of National Adult Reading Test. Psychiatry Clin Neurosci, 2006; 60: 332-339.

19) Fletcher PC, Frith CD, Grasby PM, Friston KJ, Dolan RJ: Local and distributed effects of apomorphine on frontotemporal function in acute unmedicated schizophrenia. J Neurosci, 1996; 16: 7055-7062.

20) Gaillard WD, Hertz-Pannier L, Mott SH, Barnett AS, LeBihan D, Theodore WH: Functional anatomy of cognitive development: fMRI of verbal fluency in children and adults. Neurology, 2000; 54: 180-185.

21) Goethals I, Audenaert K, Jacobs F, et al: Cognitive neuroactivation using SPECT and the Stroop Colored Word Test in patients with diffuse brain injury. J Neurotrauma, 2004; 21: 1059-1069.

22) Carter CS, Mintun M, Cohen JD: Interference and facilitation effects during selective attention: an $\mathrm{H} 215 \mathrm{O}$ PET study of Stroop task performance. Neuroimage, 1995; 2: 264-272.

23) Ohnuma T, Sakai Y, Maeshima H, et al: No correlation between plasma NMDA-related glutamatergic amino acid levels and cognitive function in medicated patients with schizophrenia. Int J Psychiatry Med, 2012; 44: 1727.

24) Guan F, Wei S, Zhang C, et al: A population-based association study of $2 \mathrm{q} 32.3$ and $8 \mathrm{q} 21.3$ loci with schizophrenia in Han Chinese. J Psychiatr Res, 2013; 47: 712-717.
25) Schizophrenia Psychiatric Genome-Wide Association Study (GWAS) Consortium: Genome-wide association study identifies five new schizophrenia loci. Nat Genet, 2011; 43: 969-976.

26) Ng MY, Levinson DF, Faraone SV, et al: Meta-analysis of 32 genome-wide linkage studies of schizophrenia. Mol Psychiatry, 2009; 14: 774-785.

27) Ripke S, O'Dushlaine C, Chambert K, et al: Genomewide association analysis identifies 13 new risk loci for schizophrenia. Nat Genet, 2013; 45: 1150-1159.

28) DeLisi LE, Shaw SH, Crow TJ, et al: A genome-wide scan for linkage to chromosomal regions in 382 sibling pairs with schizophrenia or schizoaffective disorder. Am J Psychiatry, 2002; 159: 803-812.

29) Fujino H, Sumiyoshi C, Sumiyoshi T, et al: Performance on the Wechsler Adult Intelligence Scale-III in Japanese patients with schizophrenia. Psychiatry Clin Neurosci, 2014; 68: 534-541.

30) Kremen WS, Seidman LJ, Faraone SV, et al: Intelligence quotient and neuropsychological profiles in patients with schizophrenia and in normal volunteers. Biol Psychiatry, 2001; 50: 453-462.

31) Wilk CM, Gold JM, McMahon RP, Humber K, Iannone $\mathrm{VN}$, Buchanan RW: No, it is not possible to be schizophrenic yet neuropsychologically normal. Neuropsychology, 2005; 19: 778-786.

32) Kremen WS, Seidman LJ, Faraone SV, Tsuang MT: IQ decline in cross-sectional studies of schizophrenia: methodology and interpretation. Psychiatry Res, 2008; 158: 181-194.

33) Ohi K, Sumiyoshi C, Fujino H, et al: A Brief Assessment of Intelligence Decline in Schizophrenia As Represented by the Difference between Current and Premorbid Intellectual Quotient. Front Psychiatry, 2017; 8: 293.

34) Weickert TW, Goldberg TE, Gold JM, Bigelow LB, Egan MF, Weinberger DR: Cognitive impairments in patients with schizophrenia displaying preserved and compromised intellect. Arch Gen Psychiatry, 2000; 57: 907-913.

35) Weinberg D, Lenroot R, Jacomb I, et al: Cognitive Subtypes of Schizophrenia Characterized by Differential Brain Volumetric Reductions and Cognitive Decline. JAMA Psychiatry, 2016; 73: 1251-1259.

36) Wells R, Swaminathan V, Sundram S, et al: The impact of premorbid and current intellect in schizophrenia: cognitive, symptom, and functional outcomes. NPJ Schizophr, 2015; 1: 15043.

37) Hodge MA, Siciliano D, Withey P, et al: A randomized controlled trial of cognitive remediation in schizophrenia. Schizophr Bull, 2010; 36: 419-427.

38) Wykes T, Huddy V, Cellard C, McGurk SR, Czobor P: A meta-analysis of cognitive remediation for schizophrenia: methodology and effect sizes. Am J Psychiatry, 2011; 168: 472-485.

39) Minzenberg MJ, Poole JH, Benton C, Vinogradov S: Association of anticholinergic load with impairment of complex attention and memory in schizophrenia. Am J Psychiatry, 2004; 161: 116-124.

40) Hindmarch I: Cognitive toxicity of pharmacotherapeutic agents used in social anxiety disorder. Int J Clin Pract, 2009; 63: 1085-1094.

41) Woodward ND, Duffy B, Karbasforoushan H: Prefrontal 
cortex activity during response selection predicts processing speed impairment in schizophrenia. J Int Neuropsychol Soc, 2013; 19: 782-791.

42) Srikantan V, Zou Z, Petrovics G, et al: PCGEM1, a prostate-specific gene, is overexpressed in prostate cancer. Proc Natl Acad Sci U S A, 2000; 97: 12216-12221.

43) Goes FS, McGrath J, Avramopoulos D, et al: Genomewide association study of schizophrenia in Ashkenazi Jews. Am J Med Genet B Neuropsychiatr Genet, 2015; 168: $649-659$.

44) Li Z, Chen J, Yu H, et al: Genome-wide association analysis identifies 30 new susceptibility loci for schizophrenia. Nat Genet, 2017; 49: 1576-1583.

45) Schizophrenia Working Group of the Psychiatric Genomics Consortium: Biological insights from 108 schizophrenia-associated genetic loci. Nature, 2014; 511: 421-427.

46) Ohi K, Hashimoto R, Yamamori H, et al: The impact of the genome-wide supported variant in the cyclin M2 gene on gray matter morphology in schizophrenia. Behav Brain Funct, 2013; 9: 40.

47) Horie M, Mitsumoto Y, Kyushiki H, et al: Identification and characterization of TMEFF2, a novel survival factor for hippocampal and mesencephalic neurons. Genomics, 2000; 67: 146-152.

48) Kanemoto N, Horie M, Omori K, et al: Expression of TMEFF1 mRNA in the mouse central nervous system: precise examination and comparative studies of TMEFF1 and TMEFF2. Brain Res Mol Brain Res, 2001; 86: $48-55$.

49) Rietveld CA, Medland SE, Derringer J, et al: GWAS of 126,559 individuals identifies genetic variants associated with educational attainment. Science, 2013; 340: 14671471.

50) Martin NW, Medland SE, Verweij KJ, et al: Educational attainment: a genome wide association study in 9538 Australians. PLoS One, 2011; 6: e20128.

51) Davies G, Marioni RE, Liewald DC, et al: Genome-wide association study of cognitive functions and educational attainment in UK Biobank (N=112 151). Mol Psychiatry, 2016; 21: 758-767.

52) Le Hellard S, Wang Y, Witoelar A, et al: Identification of Gene Loci That Overlap Between Schizophrenia and Educational Attainment. Schizophr Bull, 2017; 43: 654664. 